\title{
Communication Intervention
}

National Cancer Institute

\section{Source}

National Cancer Institute. Communication Intervention. NCI Thesaurus. Code C116520.

An act of intercession by an outside party for the purpose of clarifying or improving the exchange of information between individuals, such as between physician and patient or by an individual with a communication disorder. 